\title{
AN ARCHIVAL STUDY OF HST OBSERVATIONS OF HER X-1/HZ HER
}

\author{
F. H. CHENG ${ }^{1}$, S. D. VRTILEK ${ }^{1,2}$, J. C. RAYMOND ${ }^{2}$ \\ 1. University of Maryland, USA \\ 2. Harvard-Smithsonian Center for Astrophysics, USA
}

\section{Introduction}

Her X-1 is an X-ray pulsar with a rotation period of $1.24 \mathrm{~s}$ and a binary period of $1.7 \mathrm{~d}$ (Tananbaum et al. 1972). The $1.7 \mathrm{~d}$ variations in optical and ultraviolet flux are attributed to X-ray heating of the companion star and disk (e.g. Howarth \& Wilson 1983, hereafter HW83). The system displays a $35 \mathrm{~d}$ period, attributed to the effects of a tilted, precessing, accretion disk. Optical and ultraviolet flux variations continue unchanged throughout. This work is motivated by the following reasons:

- The observed IUE spectra have significantly flatter slopes than those predicted by previous models (e.g. HW83).

- The observed strength of the Balmer jump is anomalously low compared to that expected for a normal B star (Anderson et al. 1994).

- HST observations obtained by Anderson et al. (1994) in order to study emission lines have yielded high quality spectra of the continuum emission from HZ Her, enabling for the first time detailed model fitting efforts.

\section{Model}

- Adopt the system geometry of HW83 and X-ray heating code described in Vrtilek et al. $(1990,1991)$.

- Assume both disk and star have an albedo of $50 \%$.

- Estimate $E(B-V)<0.05$ from the lack of a $2200 \AA$ absorption feature.

- Use $L_{\mathrm{x}}=0.5 G M \dot{M} / r_{1}$, where $r_{1}$ is the radius of the neutron star.

- Calculate the temperature at star and disk surfaces due to X-ray heating. Use IUE and Kitt Peak stellar fluxes (referred to as star-type spectra) for that temperature to determine UV/optical continuum flux (HW83 used model stellar atmospheres). 

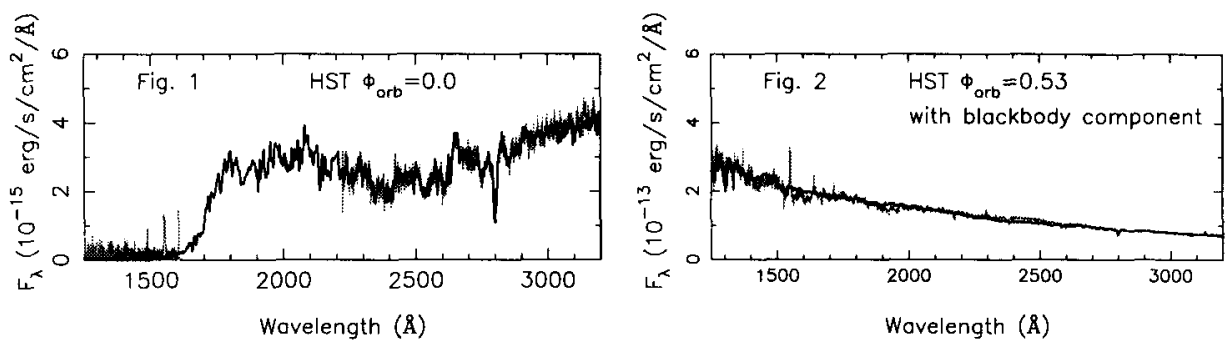

- Include blackbody component. Introduce two critical temperatures: $T_{\mathrm{sc}}$ and $T_{\mathrm{dc}}$. For a given area element in the disk and star, if the temperatures $T_{\mathrm{d}}$ and $T_{\mathrm{s}}$ are higher than $T_{\mathrm{dc}}$ or $T_{\mathrm{sc}}$, compute the flux using a blackbody, otherwise use star-type spectrum.

- Free parameters are $\dot{M}, T_{\mathrm{dc}}$ and $T_{\mathrm{sc}}, \phi_{\mathrm{orb}}, \phi_{35}$. Fixed parameters are $q, \beta$, $\theta_{\mathrm{d}}, \alpha_{\mathrm{d}}, \Delta \psi, i, a, d, r_{1}$ and $r_{2}$ (a detailed description of the model can be seen in Cheng, Vrtilek \& Raymond 1995).

\section{Results}

- At $\phi_{\text {orb }} \sim 0.0$, we obtain the average temperature of the unheated star surface $T_{\mathrm{s} 0}=8100 \pm 240 \mathrm{~K}$ (Fig. 1).

- At $\phi_{\text {orb }} \sim 0.5$, we obtain $T_{\mathrm{dc}} \sim 10000 \mathrm{~K}, T_{\text {sc }} \sim 18900 \mathrm{~K}$, and $\dot{M}=$ $(6.5 \pm 0.9) 10^{-9} \mathrm{M}_{\odot} \mathrm{yr}^{-1}$ (Fig. 2).

- Balmer Jump: see Table 1.

TABLE 1. Comparison of the Balmer Jump

\begin{tabular}{lcc}
\hline$\phi_{\text {orb }}$ & Observed BJ & Model BJ \\
\hline 0.0 & 3.0 & 2.8 \\
0.53 & 1.3 & 1.8 (no blackbody) \\
0.53 & 1.3 & 1.4 (with blackbody) \\
\hline
\end{tabular}

\section{References}

Anderson, S.F., Wachter, S., Margon, B., et al., 1994, Ap. J., 436, 319

Cheng, F.H., Vrtilek, S.D., Raymond, J.C., 1995, Ap. J., 452, 825

Howarth, I.D., Wilson, B., 1983, MNRAS, 204, 347 (HW83)

Tananbaum, H., Gursky, H., Kellogg, E.M., et al., 1972, Ap. J., 174, L143

Vrtilek, S.D., Penninx, W., Raymond, J.C., et al., 1991, Ap. J., 376, 278

Vrtilek, S.D., Raymond, J. C., Garcia, M.R., et al., 1990, A\&A, 235, 162 